\title{
Solving Hammerstein Type Integral Equation by New Discrete Adomian Decomposition Methods
}

\author{
Huda O. Bakodah ${ }^{1}$ and Mohamed Abdalla Darwish ${ }^{1,2}$ \\ ${ }^{1}$ Department of Mathematics, Sciences Faculty for Girls, King Abdulaziz University, Jeddah, Saudi Arabia \\ ${ }^{2}$ Department of Mathematics, Faculty of Science, Damanhour University, Damanhour, Egypt
}

Correspondence should be addressed to Mohamed Abdalla Darwish; dr.madarwish@gmail.com

Received 2 May 2013; Accepted 4 October 2013

Academic Editor: Metin O. Kaya

Copyright (C) 2013 H. O. Bakodah and M. A. Darwish. This is an open access article distributed under the Creative Commons Attribution License, which permits unrestricted use, distribution, and reproduction in any medium, provided the original work is properly cited.

New discrete Adomian decomposition methods are presented by using some identified Clenshaw-Curtis quadrature rules. We investigate two mixed quadrature rules one of precision five and the other of precision seven. The first rule is formed by using the Fejér second rule of precision three and Simpson 1/3 rule of precision three, while the second rule is formed by using the Fejér second rule of precision five and the Boole rule of precision five. Our methods were applied to a nonlinear integral equation of the Hammerstein type and some examples are given to illustrate the validity of our methods.

\section{Introduction}

In this paper we study the problem of approximate solutions for the nonlinear integral equations of the Hammerstein type:

$$
\begin{array}{r}
\lambda x(t)=y(t)+\int_{a}^{b} k(t, s) u(x(s)) d s, \\
\lambda \neq 0 ; \quad a \leq t \leq b .
\end{array}
$$

Nonlinear integral equations arise naturally in many applications in describing numerous real world problems. For example, it occurs in solving several problems arising in economics, engineering, and physics. One of the most important frequently investigated nonlinear integral equations is the Hammerstein integral equation (cf. [1-5]).

On the other hand, there are significant interests in applying the Adomian decomposition method (ADM) for a wide class of nonlinear equations, for example, ordinary and partial differential equations, integral equations, and integrodifferential equations; see [6-9] and references therein.

In [10], Behiry et al. introduced a discrete version of the Adomian decomposition method and applied it to (1). This method is called a discrete Adomian decomposition method (DADM). DADM arises when the quadrature rules are used to approximate the definite integrals which cannot be computed analytically. The DADM gives the numerical solution at nodes used in the quadrature rules.

Dash and Das [11, 12] used mixed quadrature rules to approximate a definite integral, namely, mixed quadrature rules blending some Fejér and Newton-Cote type rule and a mixed quadrature rule blending Clenshaw-Curtis five-point rule and Gauss-Legendre three-point rule. Behiry and other [10] applied the Simpson rule with $n$ subinterval and step size $h=(b-a) / n$. It is occasionally useful, both theoretically and practically, to have interpolatory formulas on sets of abscissas other than the equidistant set. A common choice is the set of zeros of an orthogonal polynomial.

Our main goal is to improve DADM, used by Behiry and other [10] to obtain approximate solutions of (1), by using mixed quadrature rules to approximate a definite integral. We use the advantage of the fact that the Fejér second rule of precision three and Simpson 1/3 rule of precision three to form a mixed quadrature rule of higher precision, that is, precision five. Also, we use the Fejér second rule of precision five and the Boole rule of precision five to form a mixed quadrature rule of precision seven. Our numerical examples show that our method gives approximate solutions to (1) more accurate than approximate solutions obtained in [10]. 


\section{Quadrature Rules}

In this section, we recall definitions of two quadrature rules which will be used throughout the paper, namely, ClenshawCurtis quadrature and Fejér quadrature. Clenshaw-Curtis quadrature and Fejér quadrature are based on an expansion of the integrand in terms of Chebyshev polynomials. So, let us first state some facts about Chebyshev polynomials. It is worth mentioning that Chebyshev polynomials are everywhere dense in numerical analysis [13].

Definition 1. The Chebyshev polynomial $T_{n}(x)$ of the first kind is a polynomial in $x$ of degree $n$, defined by the following relation:

$$
T_{n}(x)=\cos n \theta, \quad \text { when } x=\cos \theta .
$$

From formula (2), the zeros for $x$ in $[-1,1]$ of $T_{n}(x)$ must correspond to the zeros for $\theta$ in $[0, \pi]$ of $\cos n \theta$, so that

$$
n \theta=(2 j-1) \frac{\pi}{2}, \quad j=1,2,3, \ldots, n .
$$

Hence, the zeros of $T_{n}(x)$ are

$$
x_{j}=\cos \frac{(2 j-1) \pi}{2 n}, \quad j=1,2,3, \ldots, n .
$$

The internal extrema of $T_{n}(x)$ correspond to the extreme values of $\cos n \theta$, namely, the zeros of $\sin n \theta$, since $(d / d x) T_{n}(x)=$ $\sin n \theta / \sin \theta$. Hence, including those at $x= \pm 1$, the extrema of $T_{n}(x)$ on $[-1,1]$ are

$$
x_{j}=\cos \frac{j \pi}{n}, \quad j=1,2,3, \ldots, n .
$$

Definition 2. The Chebyshev polynomial $U_{n}(x)$ of the second kind is a polynomial in $x$ of degree $n$, defined by the following relation:

$$
U_{n}(x)=\frac{\sin (n+1) \theta}{\sin \theta}, \quad \text { when } x=\cos \theta
$$

The zeros of $U_{n}(x)$ are given by

$$
x_{j}=\cos \frac{j \pi}{n+1}, \quad j=1,2,3, \ldots, n .
$$

2.1. Clenshaw-Curtis Quadrature. Clenshaw-Curtis quadrature method proposed by Clenshaw and Curtis [14] amounts to integrating via a change of variable $x=\cos (\theta)$. The algorithm is normally expressed for integration of a function $f(x)$ over the interval $[-1,1]$; any other interval can be obtained by appropriate rescaling. For this integral, we can write

$$
\int_{-1}^{1} f(x) d x=\int_{0}^{\pi} f(\cos \theta) \sin (\theta) d \theta
$$

That is, we have transformed the problem from integrating $f(x)$ to one of integrating $f(\cos \theta) \sin \theta$. This can be performed if we know the cosine series for $f(\cos \theta)$. The reason that this is connected to the Chebyshev polynomials
$T_{j}(x)$ is that, by (2), $T_{j}(\cos \theta)=\cos (j \theta)$, and so the cosine series is really an approximation of $f(x)$ by Chebyshev polynomials:

$$
f(x)=\frac{a_{0}}{2} T_{0}(x)+\sum_{j=1}^{n} a_{j} T_{j}(x), \quad x \in[-1,1],
$$

and thus we are integrating $f(x)$ by integrating its approximate expansion in terms of Chebyshev polynomials. The evaluation points $x_{j}=\cos (j \theta / n)$ correspond to the extrema of the Chebyshev polynomial $T_{n}(x)$; see (5). The fact that such Chebyshev approximation is just a cosine series under a change of variables is responsible for the rapid convergence of the approximation as more terms $T_{j}(x)$ are included. A cosine series converges very rapidly for functions that are even, periodic, and sufficiently smooth. This is true here, since $f(\cos \theta)$ is even and periodic in $\theta$ by construction, and is $j$-times differentiable everywhere if $f(x)$ is $j$-times differentiable on $[-1,1]$.

2.2. Fejér Quadrature. Let $n \geq 2$ be a given fixed integer, and define the $(n+1)$ quadrature nodes on the interval $[-1,1]$ as the extrema of the Chebyshev polynomial $T_{n}(x)$ arguments by the boundary points:

$$
x_{j}=\cos \vartheta_{j}, \quad \vartheta_{j}=\frac{j \pi}{n}, j=0,1,2, \ldots, n .
$$

Fejér's first rule [15] is obtained by using the well-known Chebyshev points as nodes, that is, $x_{j}$ from (10) with $j=1 / 2$, $3 / 2, \ldots, n-1 / 2$, namely,

$$
R_{1} F_{n}(f)=\sum_{j=0}^{n} w_{j} f\left(x_{j}\right)=\sum_{j=0}^{n} w_{j} f\left(\cos \frac{j \pi}{n}\right),
$$

with the corresponding weights

$$
w_{j}=\frac{2}{n}\left[1-2 \sum_{m=1}^{[n / 2]} \frac{\cos \left(m \vartheta_{j+1}\right)}{4 m^{2}-1}\right], \quad j=1,2, \ldots, n-1 .
$$

Fejér's second rule [15] is obtained by omitting the nodes $x_{0}=1$ and $x_{n}=-1$ and using the interpolating polynomial of degree $n-2$. This may also be achieved by keeping the boundary points as nodes but preassigning the corresponding weights as $w_{0}=w_{n}=0$. Then Fejér's second rule is given by

$$
R_{2} F_{n}(f)=\sum_{j=0}^{n} w_{j}^{*} f\left(x_{j}^{*}\right)=\sum_{j=0}^{n} w_{j}^{*} f\left(\cos \frac{j \pi}{n+1}\right)
$$

with the corresponding weights

$$
w_{j}^{*}=\frac{4}{n} \sin \vartheta_{j} \sum_{m=1}^{[n / 2]} \frac{\sin \left[(2 m-1) \vartheta_{j}\right]}{2 m-1}, \quad j=0,2, \ldots, n .
$$




\section{DADM with New Nodes}

By applying ADM, the solution $x$ of (1) is given by the following series form:

$$
x(t)=\sum_{k=0}^{\infty} x_{k}(t),
$$

where the components $x_{k}(t), k \geq 0$, can be computed later on. We represent the nonlinear term $u(x(t))$ by the Adomian polynomials, $A_{k}(t)$, as follows:

$$
u(x(t))=\sum_{k=0}^{\infty} A_{k}\left[x_{0}(t), x_{1}(t), \ldots, x_{k}(t)\right],
$$

where $A_{k}(t)$ can be evaluated by the following formula [16]:

$$
A_{k}\left[x_{0}(t), x_{1}(t), \ldots, x_{k}(t)\right]=\frac{1}{k !} \frac{d^{k}}{d \beta^{k}}\left[u\left(\sum_{k=0}^{\infty} \beta^{k} x_{k}\right)\right]_{\beta=0} \text {. }
$$

By substituting (15) and (16) into (1), we obtain

$$
\sum_{k=0}^{\infty} x_{k}(t)=\frac{1}{\lambda} y(t)+\frac{1}{\lambda} \sum_{k=0}^{\infty} \int_{a}^{b} k(t, s) A_{k}(s) d s .
$$

Now, we can compute the components $x_{k}(t), k \geq 0$, by using the following recursive relations [17]:

$$
\begin{gathered}
x_{k+1}(t)=\frac{1}{\lambda} \int_{a}^{b} k(t, s) A_{k}(s) d s, \quad k \geq 0, \\
x_{0}(t)=\frac{y(t)}{\lambda} .
\end{gathered}
$$

It is noticed that the computation of each component $x_{k}(t)$, $k \geq 0$, requires the computation of an integral in (19). If the evaluation of integral in (19) is analytically impossible, the ADM cannot be applied. In order to use numerical integration method for integral in (19), we transform the interval $[a, b]$ into the interval $[-1,1]$ by using the transformation

$$
\tau=\frac{1}{2}[(a+b)+(b-a) t] .
$$

Now, we will make use of the following two quadrature rules.

Rule 1. Here, the construction mixed quadrature rule will be of precision five [11].

We consider the Fejér second rule of precision three:

$$
\begin{aligned}
I(f) & =\int_{-1}^{1} f(x) d x \simeq R_{2 F_{3}}(f) \\
& =\frac{2}{3}\left[f\left(\frac{1}{\sqrt{2}}\right)+f(0)+f\left(\frac{-1}{\sqrt{2}}\right)\right]
\end{aligned}
$$

and the Simpson $1 / 3$ rule of precision three:

$$
\begin{aligned}
I(f) & =\int_{-1}^{1} f(x) d x \simeq R_{S}(f) \\
& =\frac{1}{3}[f(-1)+4 f(0)+f(1)] .
\end{aligned}
$$

Then, we obtain the mixed quadrature rule of precision five for the approximate evaluation of $I(f)$; namely,

$$
\begin{aligned}
& R_{2 F_{3} S}(f) \\
& =\frac{1}{15}\left[f(-1)+8 f\left(\frac{-1}{\sqrt{2}}\right)+12 f(0)+8 f\left(\frac{1}{\sqrt{2}}\right)+f(1)\right]
\end{aligned}
$$

with a truncation error $E_{2} F_{3} S$ given by

$$
E_{2 F_{3} S}(f)=\frac{1}{37800} f^{(v i)}(0)+\cdots .
$$

Rule 2. In this rule the mixed quadrature rule will be of precision seven [11].

We consider the Fejér second rule of precision five:

$$
\begin{array}{r}
R_{2 F_{5}}(f)=\frac{2}{45}\left[7 f\left(\frac{\sqrt{3}}{2}\right)+9 f\left(\frac{1}{2}\right)+13 f(0)\right. \\
\left.+9 f\left(\frac{-1}{2}\right)+7 f\left(\frac{-\sqrt{3}}{2}\right)\right]
\end{array}
$$

and the Boole rule of precision five:

$$
\begin{aligned}
R_{B}(f)=\frac{1}{45}[ & 7 f(-1)+32 f\left(\frac{-1}{2}\right)+12 f(0) \\
+ & \left.32 f\left(\frac{1}{2}\right)+7 f(1)\right]
\end{aligned}
$$

Then, we obtain the mixed quadrature rule of precision seven for the approximate evaluation of $I(f)$; namely,

$$
\begin{aligned}
& R_{2 F_{5} B}(f) \\
& =\frac{1}{315}\left[9 f(-1)+80 f\left(\frac{-\sqrt{3}}{2}\right)+144 f\left(\frac{-1}{2}\right)+164 f(0)\right. \\
& \left.\quad+144 f\left(\frac{1}{2}\right)+80 f\left(\frac{\sqrt{3}}{2}\right)+9 f(1)\right]
\end{aligned}
$$

with a truncation error $E_{2} F_{5} B$ given by

$$
E_{2 F_{5} B}(f)=\frac{1}{140 \times 9 !} f^{(v i i i)}(0)+\cdots .
$$

\section{Numerical Examples}

In this section we apply our methods to some integral equations of the Hammerstein type. These examples show the efficient and accuracy of our methods. The tables show computed absolute error:

$$
\left|e_{m}(t)\right|=\left|x_{\text {exact }}(t)-x_{\text {app. }}(t)\right|,
$$

where $m$ is the number of the components $x_{1}, x_{2}, \ldots, m$. The computations associated with examples are performed using Mathematica 6. 
TABLE 1: The effect of $m$ in the absolute error at $n=3$.

\begin{tabular}{lccc}
\hline \multicolumn{4}{c}{$R_{2 F_{3}}$} \\
$t$ & $\left|e_{3}(t)\right|$ & $\left|e_{4}(t)\right|$ & $\left|e_{5}(t)\right|$ \\
\hline 0.14645 & $4.93549 \times 10^{-3}$ & $4.43266 \times 10^{-3}$ & $4.26484 \times 10^{-3}$ \\
0.50000 & $5.251397 \times 10^{-3}$ & $4.71637 \times 10^{-3}$ & $4.53780 \times 10^{-3}$ \\
0.85355 & $8.38783 \times 10^{-3}$ & $7.53327 \times 10^{-3}$ & $7.24806 \times 10^{-6}$ \\
\hline
\end{tabular}

TABLE 2: The effect of $m$ in the absolute error at $n=5$.

\begin{tabular}{lccc}
\hline \multicolumn{3}{c}{$R_{2 F_{5} s}$} \\
$t$ & $\left|e_{3}(t)\right|$ & $\left|e_{4}(t)\right|$ & $\left|e_{5}(t)\right|$ \\
\hline 0.00000 & $1.68344 \times 10^{-3}$ & $9.07620 \times 10^{-4}$ & $6.12675 \times 10^{-4}$ \\
0.14645 & $1.68421 \times 10^{-3}$ & $9.08038 \times 10^{-4}$ & $6.12957 \times 10^{-4}$ \\
0.50000 & $1.79201 \times 10^{-3}$ & $9.66157 \times 10^{-4}$ & $6.52190 \times 10^{-4}$ \\
0.85355 & $2.86231 \times 10^{-3}$ & $1.54320 \times 10^{-3}$ & $1.04172 \times 10^{-4}$ \\
1.00000 & $4.57606 \times 10^{-3}$ & $2.46717 \times 10^{-3}$ & $1.66540 \times 10^{-3}$ \\
\hline
\end{tabular}

TABLE 3: The effect of $m$ in the absolute error at $n=7$.

\begin{tabular}{lccc}
\hline \multicolumn{4}{c}{$R_{2 F_{7} B}$} \\
$t$ & $\left|e_{3}(t)\right|$ & $\left|e_{4}(t)\right|$ & $\left|e_{5}(t)\right|$ \\
\hline 0.00000 & $1.35339 \times 10^{-3}$ & $2.35630 \times 10^{-4}$ & $1.91978 \times 10^{-6}$ \\
0.06699 & $1.35388 \times 10^{-3}$ & $2.35636 \times 10^{-4}$ & $1.91988 \times 10^{-6}$ \\
0.25000 & $1.35916 \times 10^{-3}$ & $2.36553 \times 10^{-4}$ & $1.92729 \times 10^{-6}$ \\
0.50000 & $1.44117 \times 10^{-3}$ & $2.50828 \times 10^{-4}$ & $2.04359 \times 10^{-6}$ \\
0.75000 & $1.85770 \times 10^{-3}$ & $3.23330 \times 10^{-4}$ & $2.63429 \times 10^{-6}$ \\
0.93301 & $2.88530 \times 10^{-3}$ & $5.02733 \times 10^{-4}$ & $4.09596 \times 10^{-6}$ \\
1.00000 & $3.00680 \times 10^{-3}$ & $6.40511 \times 10^{-4}$ & $5.21850 \times 10^{-6}$ \\
\hline
\end{tabular}

TABLE 4: The effect of $m$ in the absolute error at $n=8$.

\begin{tabular}{lccc}
\hline$t$ & $\left|e_{3}(t)\right|$ & $\left|e_{4}(t)\right|$ & $\left|e_{5}(t)\right|$ \\
\hline 0.00000 & $1.1673 \times 10^{-3}$ & $3.2941 \times 10^{-4}$ & $3.0618 \times 10^{-6}$ \\
0.25000 & $1.1718 \times 10^{-3}$ & $3.3070 \times 10^{-4}$ & $3.0738 \times 10^{-6}$ \\
0.50000 & $1.2426 \times 10^{-3}$ & $3.5066 \times 10^{-4}$ & $3.2593 \times 10^{-6}$ \\
0.75000 & $1.6017 \times 10^{-3}$ & $4.5201 \times 10^{-4}$ & $4.2014 \times 10^{-6}$ \\
1.00000 & $3.1730 \times 10^{-3}$ & $8.9543 \times 10^{-4}$ & $8.3229 \times 10^{-6}$ \\
\hline
\end{tabular}

Example 1. Consider the nonlinear integral equation:

$$
\begin{aligned}
10 x(t)= & 10 t-\frac{1}{4}(e-1) \exp \left(t^{4}\right) \\
& +\int_{0}^{1} \exp \left(s^{4}+t^{4}\right)(x(s))^{3} d s .
\end{aligned}
$$

Here, $\lambda=10, y(t)=10 t-(1 / 4)(e-1) \exp \left(t^{4}\right), k(t, s)=$ $\exp \left(s^{4}+t^{4}\right)$, and $u(x(t))=(x(s))^{3}$. Equation (30) has an exact solution $x_{e}(t)=t,[18]$.

Let $x_{0}(t)=y(t) / \lambda=t-(1 / 40)(e-1) \exp \left(t^{4}\right)$.

Tables 1, 2, and 3 the results of our example is stated while Table 4, the results obtained in [10] are shown.
TABLE 5: The effect of $m$ in the absolute error at $n=3$.

\begin{tabular}{lccc}
\hline \multicolumn{4}{c}{$R_{2 F_{3}}$} \\
$t$ & $\left|e_{3}(t)\right|$ & $\left|e_{4}(t)\right|$ & $\left|e_{5}(t)\right|$ \\
\hline 0.14645 & $3.02305 \times 10^{-4}$ & $3.24491 \times 10^{-4}$ & $3.26736 \times 10^{-4}$ \\
0.50000 & $1.64103 \times 10^{-4}$ & $1.82928 \times 10^{-4}$ & $1.84830 \times 10^{-4}$ \\
0.85355 & $5.60069 \times 10^{-6}$ & $1.87365 \times 10^{-5}$ & $2.00742 \times 10^{-5}$ \\
\hline
\end{tabular}

TABLE 6: The effect of $m$ in the absolute error at $n=5$.

\begin{tabular}{lccc}
\hline \multicolumn{3}{c}{$R_{2 F_{5} S}$} \\
$t$ & $\left|e_{3}(t)\right|$ & $\left|e_{4}(t)\right|$ & $\left|e_{5}(t)\right|$ \\
\hline 0.00000 & $3.48603 \times 10^{-5}$ & $1.19222 \times 10^{-5}$ & $9.59700 \times 10^{-6}$ \\
0.14645 & $3.69623 \times 10^{-5}$ & $1.46203 \times 10^{-5}$ & $1.23539 \times 10^{-5}$ \\
0.50000 & $3.87246 \times 10^{-5}$ & $1.97467 \times 10^{-5}$ & $1.78171 \times 10^{-5}$ \\
0.85355 & $3.56966 \times 10^{-5}$ & $2.24303 \times 10^{-5}$ & $2.16749 \times 10^{-5}$ \\
1.00000 & $3.31078 \times 10^{-5}$ & $2.27365 \times 10^{-5}$ & $2.16749 \times 10^{-5}$ \\
\hline
\end{tabular}

TABLE 7: The effect of $m$ in the absolute error at $n=7$.

\begin{tabular}{lccc}
\hline \multicolumn{3}{c}{$R_{2 F_{7} B}$} \\
$t$ & $\left|e_{3}(t)\right|$ & $\left|e_{4}(t)\right|$ & $\left|e_{5}(t)\right|$ \\
\hline 0.00000 & $2.56882 \times 10^{-5}$ & $4.05683 \times 10^{-7}$ & $1.23148 \times 10^{-7}$ \\
0.06699 & $2.54489 \times 10^{-5}$ & $3.99928 \times 10^{-7}$ & $1.19841 \times 10^{-7}$ \\
0.25000 & $2.42181 \times 10^{-5}$ & $3.75164 \times 10^{-7}$ & $1.08116 \times 10^{-7}$ \\
0.50000 & $2.12422 \times 10^{-5}$ & $3.21320 \times 10^{-7}$ & $8.63619 \times 10^{-8}$ \\
0.75000 & $1.69456 \times 10^{-5}$ & $2.47497 \times 10^{-7}$ & $5.92382 \times 10^{-8}$ \\
0.93301 & $1.31145 \times 10^{-5}$ & $1.83399 \times 10^{-7}$ & $3.69417 \times 10^{-8}$ \\
1.00000 & $1.15954 \times 10^{-5}$ & $1.58286 \times 10^{-7}$ & $2.84312 \times 10^{-8}$ \\
\hline
\end{tabular}

TABLE 8: The effect of $m$ in the absolute error at $n=8$.

\begin{tabular}{lccc}
\hline$t$ & $\left|e_{3}(t)\right|$ & $\left|e_{4}(t)\right|$ & $\left|e_{5}(t)\right|$ \\
\hline 0.00000 & $3.4816 \times 10^{-3}$ & $2.7837 \times 10^{-4}$ & $5.1932 \times 10^{-5}$ \\
0.25000 & $3.2659 \times 10^{-3}$ & $2.6006 \times 10^{-4}$ & $5.3149 \times 10^{-5}$ \\
0.50000 & $2.8471 \times 10^{-3}$ & $2.2559 \times 10^{-4}$ & $5.1061 \times 10^{-5}$ \\
0.75000 & $2.2513 \times 10^{-3}$ & $1.7709 \times 10^{-4}$ & $4.5798 \times 10^{-5}$ \\
1.00000 & $1.5155 \times 10^{-3}$ & $1.1758 \times 10^{-4}$ & $3.7688 \times 10^{-5}$ \\
\hline
\end{tabular}

Example 2. Consider the nonlinear integral equation:

$$
\begin{aligned}
20 x(t)= & 20 t+\cos (e+t)-\cos (1+t) \\
& +\int_{0}^{1} \exp (x(s)) \sin \left(t+e^{s}\right) d s .
\end{aligned}
$$

Here, $\lambda=20, y(t)=20 t+\cos (e+t)-\cos (1+t), k(t, s)=$ $\sin \left(t+e^{s}\right)$, and $u(x(t))=\exp (x(s))$. Equation (31) has an exact solution $x_{e}(t)=t$, [18].

Let $x_{0}(t)=y(t) / \lambda=t+(1 / 20) \cos (e+t)-(1 / 20) \cos (1+t)$.

The results of this example represents in Tables 5,6 , and 7 while Table 8 represents the results given in [10].

\section{Conclusions}

In this paper, we use a new discrete Adomian decomposition method (NADM) to obtain numerical solutions of integral equations of the Hammerstein type. Our obtained 
results indicate that our method is a remarkably successful numerical technique for solving integral equations of the Hammerstein type. We make a comparison between our results in Tables 3 and 7 and results obtained in [10] (see Tables 4 and 8), and we found that our results are more accurate than results obtained in [10].

\section{References}

[1] J. Appell and C.-J. Chen, "How to solve Hammerstein equations," Journal of Integral Equations and Applications, vol. 18, no. 3, pp. 287-296, 2006.

[2] K. Deimling, Nonlinear Functional Analysis, Springer, Berlin, Germany, 1985.

[3] A. A. El-Bary, "Sobolev's method for Hammerstein integral equations," Mathematical \& Computational Applications, vol. 11, no. 2, pp. 91-94, 2006.

[4] X.-L. Liu, "On a nonlinear Hammerstein integral equation with a parameter," Nonlinear Analysis. Theory, Methods \& Applications, vol. 70, no. 11, pp. 3887-3893, 2009.

[5] D. O’Regan and M. Meehan, Existence Theory For Nonlinear Integral and Integrodierential Equations, Kluwer Academic Publishers, Dordrecht, The Netherlands, 1998.

[6] H. L. Arora and F. I. Abdelwahid, "Solution of non-integer order differential equations via the Adomian decomposition method," Applied Mathematics Letters, vol. 6, no. 1, pp. 21-23, 1993.

[7] M. M. Hosseini and H. Nasabzadeh, "Modified Adomian decomposition method for specific second order ordinary differential equations," Applied Mathematics and Computation, vol. 186, no. 1, pp. 117-123, 2007.

[8] D. Kaya, "The use of Adomian decomposition method for solving a specific nonlinear partial differential equations," Bulletin of the Belgian Mathematical Society, vol. 9, no. 3, pp. 343-349, 2002.

[9] S. Shah, A. Shaikh, and S. H. Sandilo, "Modied decomposition method for nonlinear Volterra-Fredholm integrodierential equation," Journal of Basic \& Applied Sciences, vol. 6, no. 1, pp. 13-16, 2010.

[10] S. H. Behiry, R. A. Abd-Elmonem, and A. M. Gomaa, "Discrete Adomian decomposition solution of nonlinear Fredholm integral equation," Ain Shams Engineering Journal, vol. 1, no. 1, pp. 97-101, 2010.

[11] R. B. Dash and D. Das, "Identification of some Clenshaw-Curtis quadrature rules as mixed quadrature of Fejer and NewtonCote type of rules," International Journal of Mathematical Sciences \& Applications, vol. 1, no. 3, pp. 1493-1496, 2011.

[12] R. B. Dash and D. Das, "A mixed quadrature rule by blending Clenshaw-Curtis and Gauss-Legendre quadrature rules for approximation of real definite integrals in adaptive environment," IMECS, vol. 2011, pp. 202-205, 2011.

[13] J. C. Mason and D. C. Handscomb, Chebyshev Polynomials, A CRC Press, New York, NY, USA, 2003.

[14] C. W. Clenshaw and A. R. Curtis, "A method for numerical integration on an automatic computer," Numerische Mathematik, vol. 2, pp. 197-205, 1960.

[15] J. Waldvogel, "Fast construction of the Fejér and ClenshawCurtis quadrature rules," Numerical Mathematics, vol. 46, no. 1, pp. 195-202, 2006.

[16] G. Adomian, Solving Frontier Problems of Physics: The Decomposition Method, vol. 60 of Fundamental Theories of Physics,
Kluwer Academic Publishers Group, Dordrecht, The Netherlands, 1994, With a preface by Yves Cherruault.

[17] A.-M. Wazwaz, A First Course in Integral Equations, World Scientific Publishing, Singapore, 1997.

[18] A. J. Jerri, Introduction to Integral Equations with Applications, John Wiley \& Sons, 2nd edition, 1999. 


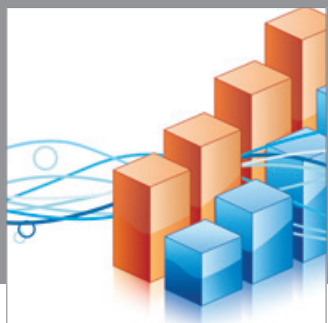

Advances in

Operations Research

mansans

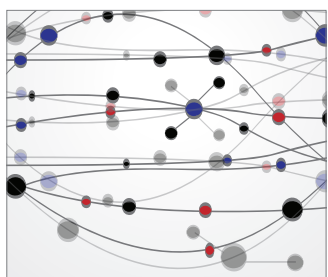

The Scientific World Journal
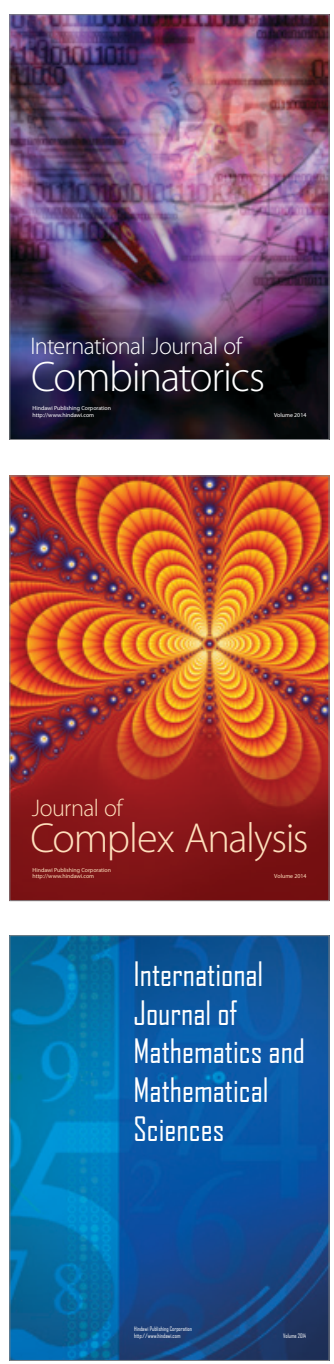
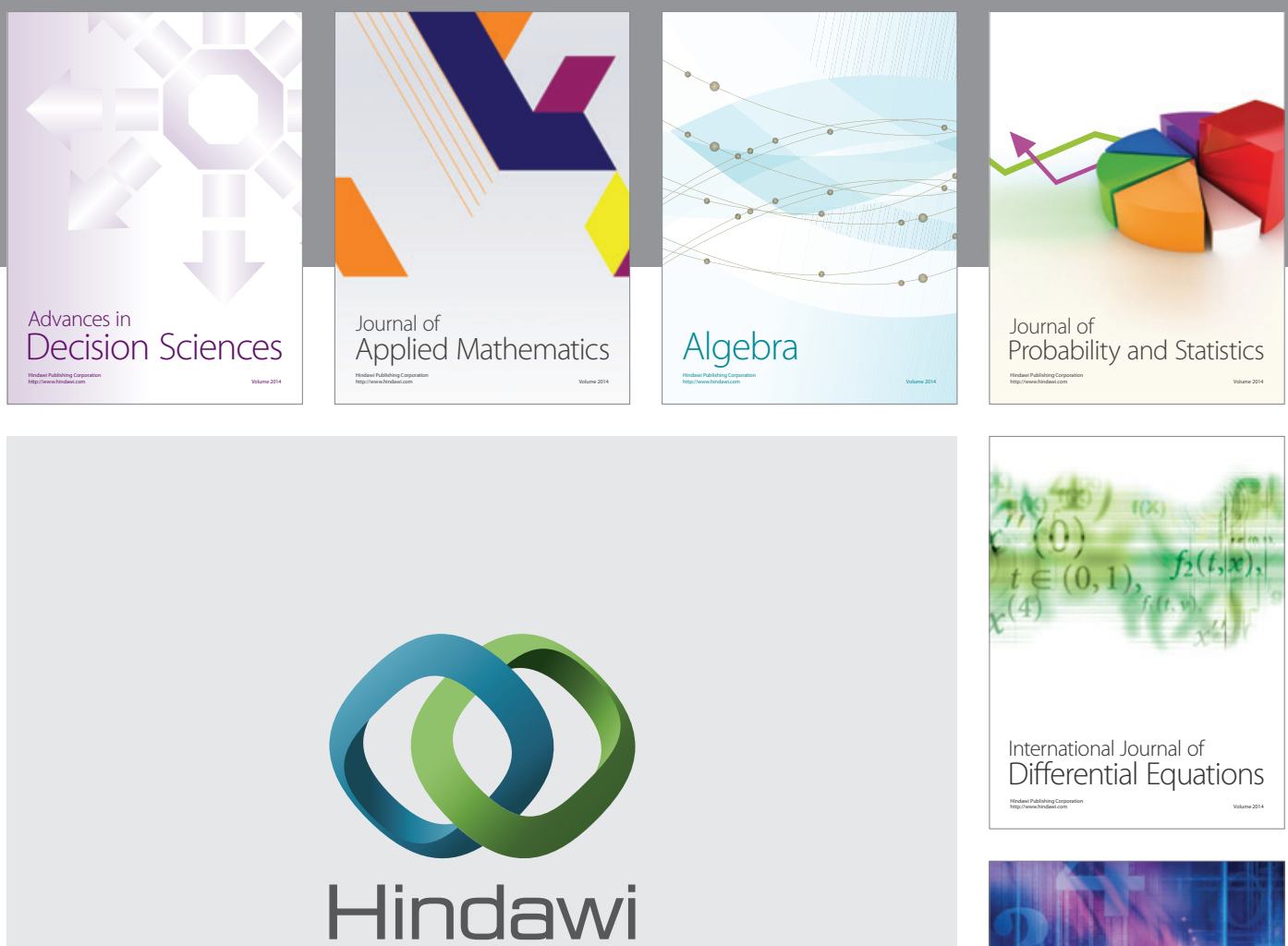

Submit your manuscripts at http://www.hindawi.com
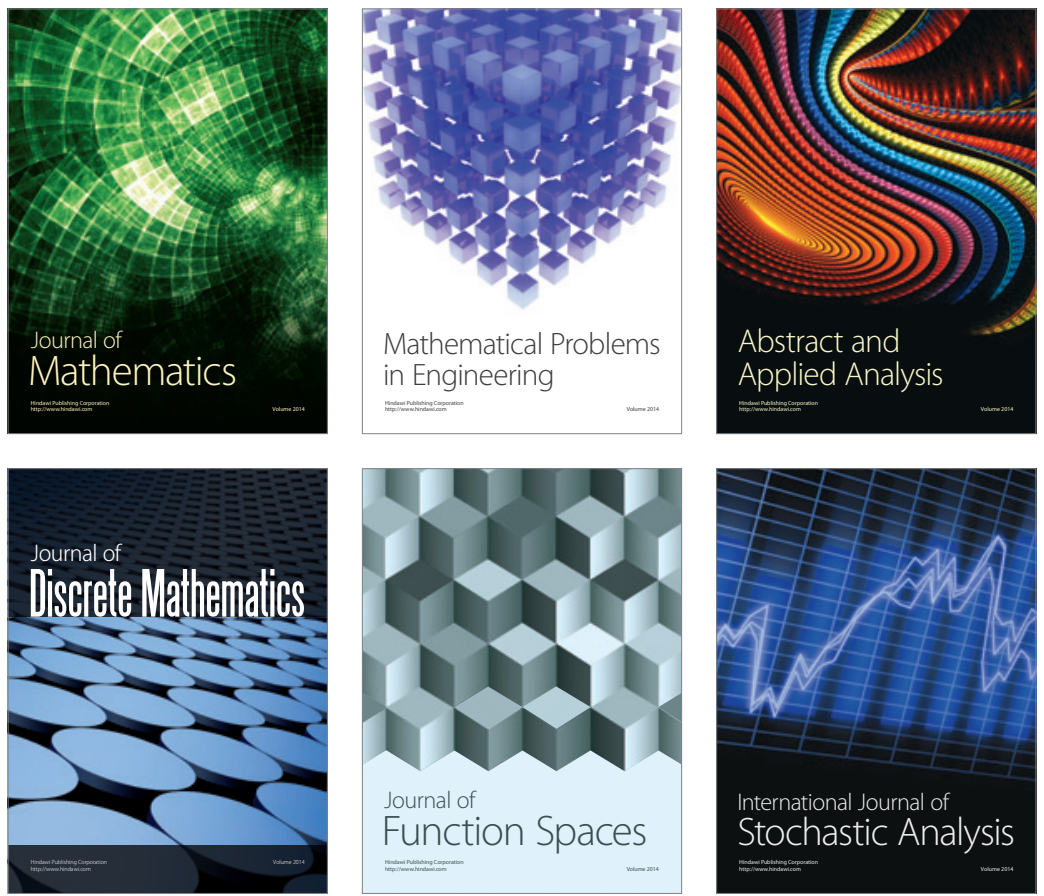

Journal of

Function Spaces

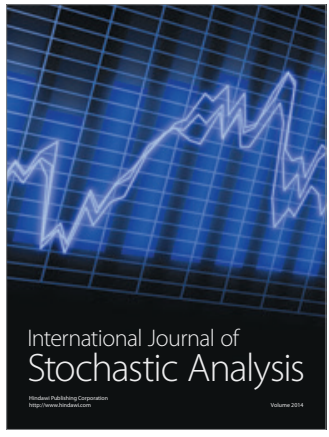

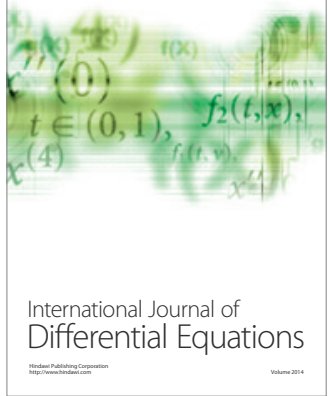
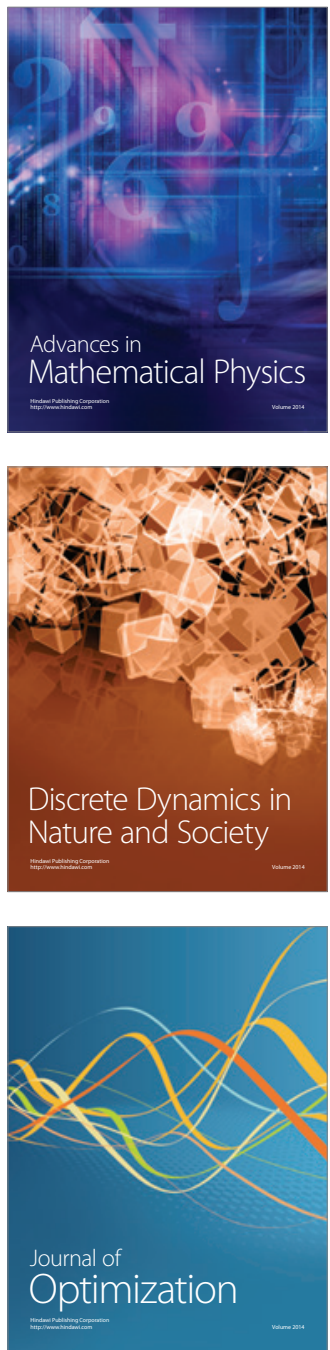